\title{
SARS-CoV-2 Infection: Paving Way for Sleep Disorders in Long Term!
}

\author{
Ravi Gupta ${ }^{1}$ (D) Seithikurippu R. Pandi-Perumal ${ }^{2}$ (])
}

Published online: 17 May 2021

(c) The Author(s), under exclusive licence to Springer Nature Singapore Pte Ltd. 2021

COVID-19 pandemic has compelled health policymakers to think of health issues from a new perspective. While on one hand, this pandemic led to the exhaustion of health resources in many geographical areas, on the other hand, this infectious disease affected the provision of medical care to patients with other medical disorders. In addition, several studies have suggested that despite being primarily a respiratory virus, SARS-CoV-2 also influences emotions, feelings, behavior, and sleep, all functions governed by the brain, leading to "mental health" consequences.

Many studies have been conducted to assess the impact of the COVID-19 pandemic on mental health and sleep disorders. Based on the findings of these studies, recently systematic reviews and meta-analyses were also published [1-5]. These papers reported a higher prevalence of anxiety, depression, and poor sleep quality among the general population, among medical healthcare workers including physicians and nurses, and among subjects suffering from COVID-19 infection, compared to population prevalence [1-5].

Deng et al. [1], in a meta-analysis where most of the included studies were from China reported that the prevalence of poor sleep quality and insomnia among patients having COVID-19 was 34\%. Although studies included in this meta-analysis used different methods to assess sleep quality and insomnia, ranging from validated questionnaires such as Pittsburgh Sleep Quality Index (PSQI) and Insomnia Severity Index (ISI) to face-to-face interviews, still the prevalence was greater than population prevalence. However, another meta-analysis, which better represented other geographical areas than the study mentioned earlier, reported that the prevalence of sleep disorders among patients having COVID-19 infection was as high as 75\% [3]. Prevalence of sleep disturbance was lower in general population and

Ravi Gupta

sleepdoc.ravi@gmail.com

1 Department of Psychiatry, Division of Sleep Medicine, All India Institute of Medical Sciences, Rishikesh 249203, India

2 Somnogen Canda Inc., College Street, Toronto, ON, Canada healthcare workers with one-third of people from the general population and a similar proportion of healthcare workers reported sleep disturbance or insomnia [3]. Interestingly, another piece of evidence suggested that the prevalence of disturbed sleep increased over time among health care workers during the pandemic [6]. Taken together, available data suggests that prevalence of disturbed sleep has risen in general population as well as healthcare workers during COVID-19 pandemic, but has gone out of proportion among persons infected with SARS-CoV-2 virus.

However, so far only cross-sectional studies have been conducted which makes it difficult to ascertain if these effects are temporary or chronic. If these are temporary, it could be attributed to many psychosocial factors as described in these papers [1-5]. These factors includes, but not limited to stress, phobia (fear of unknown disease), resource constraints (people, equipment, and facilities), witnessing deaths, pain, medication effects, distorted perception, and a plethora of misinformation shared on social media. However, there are certain issues considering these factors as etiological in the pathogenesis of sleep disorders. First, while the whole population was exposed to these factors, only a proportion of them reported depression, anxiety, and insomnia. According to one school of thought, this could be explained from the perspective of the stress-diathesis model, which proposes that people who developed insomnia, depression, and anxiety had a biological vulnerability that uncovered symptoms in the presence of environmental perturbation (COVID-19) [7]. But, given other evidence presented below, it appears an over-simplified explanation.

Recent preclinical as well as clinical studies have shown that the SARS-CoV-2 virus has significant neurological effects [8]. These effects are thought to be induced by a combination of several factors that include direct cortical involvement by the virus, cerebrovascular changes, neural involvement after systemic inflammation, and the pathological effect of the virus to alter functions of peripheral organs (e.g., lung and liver) that can be detrimental to the brain in turn [8]. The virus also reaches the hypothalamus and brainstem, where most of the nuclei of ascending 
reticular activating system are present. These areas play a major role to regulate the sleep-wake cycle and cardiorespiratory physiology besides influencing emotions and behavior [9]. SARS-CoV-2 infection also invokes systemic inflammatory response that can increase the permeability of the blood-brain barrier besides direct invasion of various neuronal structures through the olfactory route [9]. Neurotropism of virus in the areas associated with sleep-wake regulation in association with increased permeability of blood-brain barrier (that allows entry of systemic inflammatory markers in the brain) perturb sleep-wake phenomenon leading to insomnia or poor quality sleep.

Angiotensin-converting enzyme 2 (ACE-2) receptors are required for the entry of the SARS-CoV-2 virus into the cell. These receptors are present in the cells of several areas in the brain, e.g., brainstem, circumventricular organ, subfornical area, paraventricular nucleus, and rostroventrolateral medulla [10]. These areas play an important role in respiratory and cardiovascular regulation. This could be a reason behind the observed higher intensive care unit admission and mortality among patients having obstructive sleep apnea who contract SARS-CoV-2 infection [11]. Further, the presence of ACE-2 receptors in the brainstem area that regulate respiration could also invoke sleep-related breathing disorders, in particular, among patients of obstructive sleep apnea who have recovered from COVID-19. Lastly, invasion of the central nervous system has not only short-term but also longterm effects that ultimately can induce neurodegeneration, making the sleep disturbances chronic [12]. Because of these reasons, sleep problems are seen not only during the acute infection but may also run a chronic course and they have a definite biological basis [8].

Considering these findings, Silva et al. [12] proposed that sleep problems during the COVID-19 pandemic are multifactorial in origin. They proposed that ongoing stress, change in circadian rhythm due to altered light exposure during the lockdown, immediate and long-term consequences of immune response to SARS-CoV-2 infection and changes in sleep/wake patterns all contribute to pave way for immediate and long-term sleep disturbances [12].

Sleep problems may appear late in the course of COVID19 , even after patients recover from the acute infection. It has been suggested that recovered patients from SARSCoV-2 infection should be followed up to look for neurodegenerative disorders such as Parkinson's disease, Multiple Sclerosis, and Narcolepsy, plausible mechanisms of which have been discussed earlier [12]. Similarly, incipient fibrotic changes in the lungs make many of the survivors to develop obstructive sleep apnea and sleep-related hypoventilation syndromes in long term [11].

Great strides have been made to understand the presentation and management of acute manifestations of SARS-CoV-2 infection so far, but there is an unmet need for addressing long-term consequences of infection. Considering current preclinical evidence, patients who are recovering from SARS$\mathrm{CoV}-2$ infection must be screened for sleep disorders, as they have the potential to develop them immediately or after recovery from acute infection. Research suggests that exercise, stress reduction techniques, chronotherapy, and nonpharmacological intervention can be useful to improve sleep. In addition, conventional wisdom suggests that practices such as yoga, pranayama, yoga nidra, meditation, use of ayurvedic, and other traditional medicines can be used as an adjuvant in achieving better sleep due to its safety profile [13].

\section{References}

1. Deng J, Zhou F, Hou W, Silver Z, Wong CY, Chang O, et al. The prevalence of depression, anxiety, and sleep disturbances in COVID-19 patients: a meta-analysis. Ann N Y Acad Sci. 2021;1486(1):90-111.

2. Hariyanto TI, Kurniawan A. Obstructive sleep apnea (OSA) and outcomes from coronavirus disease 2019 (COVID-19) pneumonia: a systematic review and meta-analysis. Sleep Med. 2021;82:47-53.

3. Jahrami H, BaHammam AS, Bragazzi NL, Saif Z, Faris M, Vitiello MV. Sleep problems during the COVID-19 pandemic by population: a systematic review and meta-analysis. J Clin Sleep Med. 2021;17(2):299-313.

4. Salari N, Khazaie H, Hosseinian-Far A, Ghasemi H, Mohammadi $\mathrm{M}$, Shohaimi S, et al. The prevalence of sleep disturbances among physicians and nurses facing the COVID-19 patients: a systematic review and meta-analysis. Global Health. 2020;16(1):92.

5. Souza LFF, Paineiras-Domingos LL, Melo-Oliveira MES, Pessanha-Freitas J, Moreira-Marconi E, Lacerda ACR, et al. The impact of COVID-19 pandemic in the quality of sleep by Pittsburgh Sleep Quality Index: a systematic review. Cien Saude Colet. 2021;26(4):1457-66.

6. Marvaldi M, Mallet J, Dubertret C, Moro MR, Guessoum SB. Anxiety, depression, trauma-related, and sleep disorders among healthcare workers during the COVID-19 pandemic: a systematic review and meta-analysis. Neurosci Biobehav Rev. 2021;126:252-64.

7. Russell G, Lightman S. The human stress response. Nat Rev Endocrinol. 2019;15(9):525-34.

8. Heneka MT, Golenbock D, Latz E, Morgan D, Brown R. Immediate and long-term consequences of COVID-19 infections for the development of neurological disease. Alzheimers Res Ther. 2020;12(1):69.

9. Schirinzi T, Landi D, Liguori C. COVID-19: dealing with a potential risk factor for chronic neurological disorders. J Neurol. 2021;268(4):1171-8.

10. Tremblay ME, Madore C, Bordeleau M, Tian L, Verkhratsky A. Neuropathobiology of COVID-19: the role for glia. Front Cell Neurosci. 2020;14:592214.

11. Miller MA, Cappuccio FP. A systematic review of COVID-19 and obstructive sleep apnoea. Sleep Med Rev. 2021;55:101382.

12. Silva E, Ono B, Souza JC. Sleep and immunity in times of COVID-19. Rev Assoc Med Bras (1992). 2020;66(suppl 2):143-7.

13. Gulia KK, Kumar VM. Importance of sleep for health and wellbeing amidst COVID-19 pandemic. Sleep Vigil. 2020;4:1-2.

Publisher's Note Springer Nature remains neutral with regard to jurisdictional claims in published maps and institutional affiliations. 